\title{
A Role for the Periaqueductal Gray in Switching Adaptive Behavioral Responses
}

\author{
M. H. Sukikara, ${ }^{1}$ S. R. Mota-Ortiz, ${ }^{2}$ M. V. Baldo, ${ }^{3}$ L. F. Felício, ${ }^{1}$ and N. S. Canteras ${ }^{2}$ \\ ${ }^{1}$ Department of Pathology, School of Veterinary Medicine, and Departments of ${ }^{2}$ Anatomy and ${ }^{3}$ Physiology and Biophysics, Institute of Biomedical Sciences, \\ University of São Paulo, CEP 05508-900 São Paulo, Brazil
}

Previous studies suggested a role for the rostral lateral periaqueductal gray (PAG) in the inhibition of maternal behavior induced by low doses of morphine in dams with previous morphine experience. In the present study, we first showed that unilateral NMDA lesions placed in this particular PAG region prevented the morphine-induced inhibition of maternal behavior in previously morphine-sensitized dams. As suggested by previous Fos data on the PAG, predatory hunting appears as a likely candidate to replace maternal behavior in the morphine-treated dams. By testing saline- and morphine-treated dams with live cockroaches only, we have presently shown that morphine challenge increased insect hunting. Moreover, morphine- and saline-treated dams were also observed in an environment containing pups and roaches. Although most of the saline-treated animals displayed active nursing and only occasionally presented insect hunting, all of the morphine-treated animals ignored the pups and avidly pursued and caught the roaches. We next questioned whether the rostral lateral PAG would be involved in this behavioral switch. Our results showed that unilateral lesions of the rostral lateral PAG, but not other parts of the PAG, partially impaired predatory hunting and restored part of the maternal response. Moreover, bilateral lesions of the rostral lateral PAG produced even more dramatic effects in inhibiting insect hunting and restoring maternal behavior. The present findings indisputably show that the rostral lateral PAG influences switching from maternal to hunting behavior in morphinetreated dams, thus supporting a previously unsuspected role for the PAG in selecting adaptive behavioral responses.

Key words: periaqueductal gray; maternal behavior; predatory hunting; behavioral selection; NMDA lesion; morphine

\section{Introduction}

The periaqueductal gray (PAG) is known to play an important role in the modulation of nociceptive sensory transmission (Besson et al., 1991; Lovick, 1993; Bandler and Shipley, 1994), regulation of the cardiovascular system (Lovick, 1993; Bandler and Shipley, 1994), vocalization (Jürgens, 1994), and in the expression of a variety of behaviors, including defensive (Bandler and Shipley, 1994; Keay and Bandler, 2001), sexual (Sakuma and Paff, 1979), and maternal behaviors (Lonstein and Stern, 1997, 1998). By and large, the PAG-related responses have been regarded as being rather stereotyped and are thought to depend on descending projections to the brainstem and spinal cord. In contrast, previous studies from our laboratory, examining the neural basis of morphine-induced inhibition of maternal behavior, brought up the suggestion of a rather unsuspected and integrative role of the PAG in influencing the selection of adaptive behavioral responses (Miranda-Paiva et al., 2003).

It is well known that administration of morphine disrupts

Received 0ct. 7, 2005; revised Jan. 18, 2006; accepted Jan. 22, 2006.

This work was supported by grants from Fundação de Amparo à Pesquisa do Estado de São Paulo (FAPESP) (01/14039-9 and 04/13849-5) and Conselho Nacional de Desenvolvimento Científico e Tecnologico (300360/ 2003-7) to N.S.C. M.H.S. and S.R.M.-0. were supported by FAPESP fellowships. We express appreciation to Brandi Rima and Kate Karelina for reviewing this manuscript.

Correspondence should be addressed to Dr. Newton Sabino Canteras, Department of Anatomy, Institute of Biomedical Sciences, University of São Paulo, Avenida Lineu Prestes, 2415, CEP 05508-900 São Paulo, SP, Brazil. E-mail: newton@icb.usp.br.

DOI:10.1523/JNEUROSCI.4279-05.2006

Copyright $\odot 2006$ Society for Neuroscience $\quad$ 0270-6474/06/262583-07\$15.00/0 maternal behavior during lactation. This effect has been often suggested to depend on opiate effects in the medial preoptic area, a region critically involved in the expression of maternal behavior (Bridges and Grimm, 1982; Grimm and Bridges, 1983; Rubin and Bridges, 1984; Mann et al., 1991). However, findings from our laboratory showed that the PAG, and not the medial preoptic region, may account for maternal behavior inhibition in previously morphine-sensitized dams challenged with low doses of morphine (3 mg/kg, i.p.). Using this pharmacological procedure, a certain level of morphine-induced activation in a particular PAG region (located in the outer half of the lateral column at the levels of the oculomotor nucleus) is apparently required to inhibit maternal behavior, and we have shown that naloxone, previously injected therein, was able to block this behavioral inhibition (Miranda-Paiva et al., 2003). The present study first examined whether this PAG region is critical for the inhibition of maternal behavior with low doses of morphine, in previously morphine-sensitized dams.

Curiously, the rostral lateral PAG activation pattern seen in response to low doses of morphine is closely similar to that occurring during predatory hunting and foraging behavior (Comoli et al., 2003; Miranda-Paiva et al., 2003), leading to an appealing supposition that the occurrence of predatory or foraging activity would be favored during morphine-induced inhibition of maternal responses. Predatory hunting has been regarded as an innate behavioral response seemingly critical for the animals' survival; indeed, rats display an innate pattern of prey hunt- 
ing similar to that one seen in other small insectivores (Eisenberg and Leyhausen, 1972; Comoli et al., 2003, 2005). We have presently observed that morphine challenge increased insect hunting in dams tested with live cockroaches only. Furthermore, when tested in an environment containing pups and roaches, morphine-treated dams expressed predatory hunting instead of maternal responsiveness. Finally, our findings support the view that this particular behavioral shift depends on the rostral lateral PAG.

Overall, the present findings bring into focus a previously unsuspected role for the PAG in selecting adaptive behavioral responses, which may be particularly relevant for improving survival chances in food-lacking or aversive environments.

\section{Materials and Methods}

Animals and housing. Subjects were adult Wistar nulliparous female rats that weighed $280-320 \mathrm{~g}$ and were $\sim 90 \mathrm{~d}$ of age at the beginning of the experiments. In all experiments, the females were timed-mated by placing them with sexually experienced males. The day when sperm was observed in vaginal lavage was designated day 1 of pregnancy. Pregnant females were housed individually in opaque polypropylene cages $(41 \times$ $34 \times 16 \mathrm{~cm}$ ) containing $\sim 1.0 \mathrm{~L}$ of medium-grade pine flakes. Food and water were available ad libitum to the animals in light-controlled (6:00 A.M. to 6:00 P.M.) and temperature-controlled $\left(23-25^{\circ} \mathrm{C}\right)$ rooms. After giving birth (day 0 of lactation), females were left with their litters (culled to eight pups on day 1 of lactation: four males and four females) until testing for maternal and/or insect-hunting behaviors on day 5 of lactation. Animals were maintained in accordance with the guidelines of the Committee on Animals of the Colégio Brasileiro de Experimentação Animal and the Committee on the Care and Use of Laboratory Animal Resources, National Research Council.

NMDA lesion. NMDA lesions were made before pregnancy, and a 2 week postsurgery period was kept before mating. For the lesion procedure, rats were deeply anesthetized with a mixture of ketamine (Vetaset; Fort Dodge Laboratory, Campinas, Brazil) and xylazine (Rompum; 1:2 v/v; $1 \mathrm{ml} / \mathrm{kg}$ body weight; Bayer, São Paulo, Brazil), and unilateral or bilateral iontophoretic deposits of a $0.15 \mathrm{~m}$ solution of NMDA (Sigma, St. Louis, MO) were placed stereotaxically into specific columns of the PAG. The following stereotaxic coordinates were used to place the iontophoretic NMDA deposits into the different PAG regions: dorsal PAG: 2.9 $\mathrm{mm}$ rostral to the interaural line, $0.2 \mathrm{~mm}$ from the midline, and $3.5 \mathrm{~mm}$ ventral to the surface of the brain; lateral PAG: $2.9 \mathrm{~mm}$ rostral to the interaural line, $0.65 \mathrm{~mm}$ from the midline, and $4.5 \mathrm{~mm}$ ventral to the surface of the brain; ventrolateral PAG: $1.4 \mathrm{~mm}$ rostral to the interaural line, $0.6 \mathrm{~mm}$ from the midline, and $5.4 \mathrm{~mm}$ ventral to the surface of the brain. NMDA deposits were made over $15 \mathrm{~min}$ through a glass micropipette (tip diameter, $20 \mu \mathrm{m}$ ) using a constant-current device (model CS3; Midgard Electronics, Canton, MA) set to deliver $-5 \mu \mathrm{A}$, with $7 \mathrm{~s}$ pulse and interpulse durations.

Experiment 1. In this experiment, we examined whether the lateral PAG is required for inhibiting maternal behavior with low doses of morphine in previously morphine-sensitized dams. Accordingly, maternal behavior testing was performed in saline-treated $(n=10)$ and morphinetreated $(n=30)$ animals. In the morphine-treated group, we tested intact dams $(n=10)$ as well as those bearing a unilateral NMDA lesion aimed at lateral $(n=10)$ or dorsal $(n=10)$ PAG columns.

Starting on day 17 of pregnancy, rats were treated for $5 \mathrm{~d}$ with saline or morphine sulfate injections ( $3.5 \mathrm{mg} / \mathrm{kg} / \mathrm{d}$, s.c.; Cristália Laboratory, São Paulo, Brazil). In the morning of postpartum day 5, dams were tested for maternal behavior. Thirty minutes before the maternal behavior testing, lactating rats received an acute injection of morphine sulfate $(3.0 \mathrm{mg} / \mathrm{kg}$, s.c.) or saline. On the test day, between 7:00 and 8:00 A.M., pups, bedding, and food pellets were removed from the home cage. Sixty minutes later, eight pups were placed back and scattered over the cage, and maternal behavior testing began. All trials were recorded on videotape for subsequent behavioral analysis. During the $30 \mathrm{~min}$ trial, we observed whether the dams expressed active nursing. Active nursing was charac- terized when a dam was arched over the pups with her legs splayed (arched nursing) or lying over the litter without her back arched and no obvious leg extension [blanket nursing (Slamberova et al., 2003)]. Other forms of maternal responses were also recorded (i.e., grooming, retrieving, and grouping the pups).

Experiment 2. Considering that morphine challenge induces in the rostral lateral PAG an activation pattern similar to that occurring during predatory hunting, in experiment 2, we compared insect hunting between saline- and morphine-treated dams. Using the same protocol as described in experiment 1 , saline-treated $(n=7)$ and morphine-treated $(n=7)$ dams were observed in an environment containing live mature cockroaches only and tested for insect hunting. On the test day (postpartum day 5), between 7:00 and 8:00 A.M., pups and bedding were removed from the cage. Sixty minutes later, five mature cockroaches (Periplaneta americana), raised for this purpose in our laboratory, were introduced into the cage for behavioral testing. During the $30 \mathrm{~min}$ trial, we observed the overall insect-hunting behavior and timed the latency to start catching the roaches (i.e., the actual time taken to grab the first prey), as well as the interval to kill and consume the five roaches.

Experiment 3. In this experiment, we investigated how insect hunting replaces maternal behavior in morphine-treated lactating rats, as well as the potential role of the PAG in switching these behavioral responses.

Using the same protocol as described in experiment 1, dams were observed in an environment containing pups and live mature cockroaches and were tested simultaneously for maternal and insect-hunting behaviors. Immediately after the pups had been placed back with the dams, five mature cockroaches (Periplaneta americana) were introduced into the cage for behavioral testing. During the $30 \mathrm{~min}$ trial, in addition to maternal responses, we observed the overall insect-hunting behaviors of the dams and timed the latency to start catching the roaches. The following experimental groups were tested: saline-treated dams $(n=13)$, morphine-treated intact dams $(n=13)$, morphine-treated dams bearing unilateral PAG lesions aimed at different PAG columns (dorsal, $n=7$; ventrolateral, $n=7$; lateral, $n=7$ ), and morphine-treated dams with bilateral lesions aimed the lateral PAG column $(n=7)$.

Unlike experiment 2, because some of the tested animals in experiment 3 did not express predatory hunting during the 30 min observation period, instead of using the actual time taken to start capturing the roaches, we chose to score these latencies for statistical analysis, as follows: 1, 0-300 s; 2, 301-600 s; 3, 601-900 s; 4, 901-1200 s; 5, 1201-1500 $\mathrm{s} ; 6,1501-1800 \mathrm{~s} ; 7,>1801 \mathrm{~s}$. This transformation allowed us to include in our analysis those animals that did not present predatory hunting within the $30 \mathrm{~min}$ observation period, thus lacking a definite measure (the seventh rank in the present analysis).

Histology. After behavioral tests, animals were terminally anesthetized with sodium pentobarbital (65 mg/kg, i.p.) and perfused transcardially with a solution of $4.0 \%$ paraformaldehyde in $0.1 \mathrm{~m}$ phosphate buffer, $\mathrm{pH}$ 7.4; the brains were removed and left overnight in a solution of $20 \%$ sucrose in $0.1 \mathrm{~m}$ phosphate buffer at $4^{\circ} \mathrm{C}$. The brains were then frozen, and $30 \mu \mathrm{m}$ sections were cut with a sliding microtome in the frontal plane collected from the caudal thalamus through the rostral levels of the pontine central gray. Sections were mounted on gelatin-coated slides and stained with thionin. The anatomical location and size of NMDA lesions were evaluated by assessing the typical neuronal cell loss and gliosis infiltration as seen in thionin-stained sections. Nissl-staining methods are widely used to examine cytotoxic lesions (Heeb and Yahr, 2000) because these methods stain both glia and neuronal cell bodies, providing a very accurate delineation of the lesion site.

Statistical analysis. In experiments 1 and 3, we scored the number of dams that displayed active nursing in each group during a $30 \mathrm{~min}$ observation period. In experiment 3 , the number of lactating rats that groomed the pups was also scored for each experimental group. Pairwise comparisons between groups were planned beforehand. The score values for active nursing or grooming from each experimental group were arranged in a $2 \times 2$ contingency table and treated by means of the Fisher's exact test.

In experiment 2, the comparison between saline- and morphinetreated dams for the latency to start capturing the prey was analyzed 

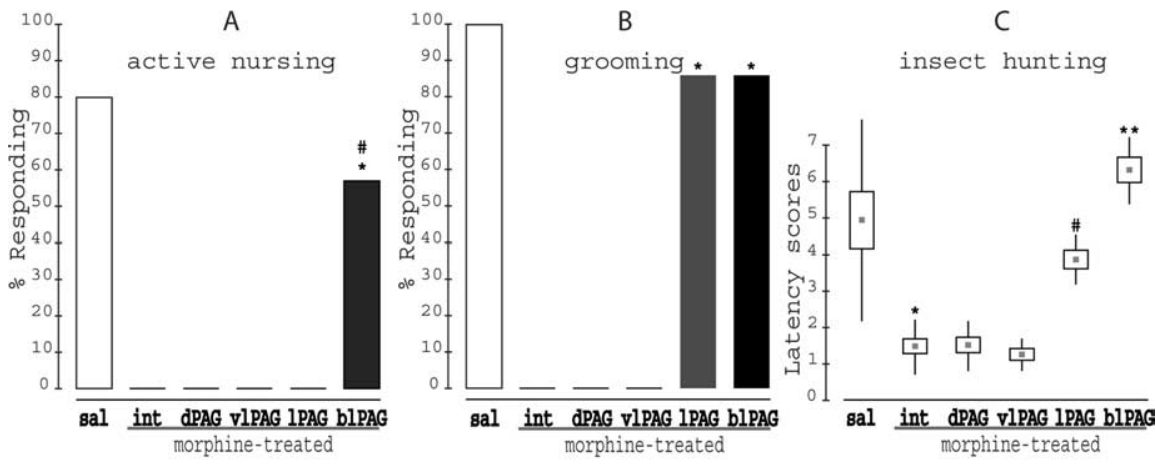

Figure 1. Experiment 3: behavioral analysis. The behavioral analysis of experiment 3 shows the percentage of animals expressing active nursing $(\boldsymbol{A})$ and grooming the pups $(\boldsymbol{B})$, as well as the latency scores to start capturing the roaches $(\boldsymbol{C})$, for the salinetreated dams (sal; $n=13$ ); the intact morphine-treated animals (int; $n=13$ ); the morphine-treated rats with unilateral lesions in the dorsal (dPAG; $n=10)$, ventrolateral (vIPAG, $n=8)$, or lateral (IPAG, $n=7)$ PAG; and the morphine-treated rats with bilateral lesions in the lateral PAG (bIPAG, $n=7$ ). ( shows the mean time taken to capture the first prey, averaged over each experimental group, after being transformed into rank-order data $(1,0-300 \mathrm{~s} ; 2,301-600 \mathrm{~s} ; 3,601-900 \mathrm{~s} ; 4,901-1200 \mathrm{~s} ; 5$, 1201-1500 s; 6, 1501-1800 s; 7,>1801 s); for illustration purposes only, these categorized data are expressed as arithmetic mean \pm SE (boxes) and arithmetic mean \pm SD (whiskers). $A,{ }^{*} p<0.005$ compared with the IPAG group, ${ }^{\#} p=0.289$ compared with the saline group; $\boldsymbol{B},{ }^{*} p<0.005$ compared with the morphine-treated intact, dPAG, or vIPAG groups; $\boldsymbol{C},{ }^{*} p=0.0085$ compared with the saline group, ${ }^{\#} p<0.002$ compared with the morphine-treated intact, dPAG, or vIPAG groups, ${ }^{* *} p=0.002$ compared with the morphine-treated IPAG group.

using a nonparametric test for independent groups (Mann-Whitney $U$ test).

In experiment 3 , the scores given to the time taken to start capturing the roaches for each experimental group were entered into a nonparametric one-way ANOVA (Kruskal-Wallis). Pairwise comparisons were planned beforehand and performed by means of the Mann-Whitney $U$ test (Sheskin, 2000). The family-wise type I error was set at $5 \%$ for all statistical procedures.

\section{Results}

In experiment 1 , low doses of morphine sulfate given on lactation day 5 inhibited maternal behavior in intact morphine-sensitized lactating rats. Immediately before the testing procedure, no clear difference in terms of general locomotor activity or any other behavioral responses was observed between saline- and morphine-treated dams. During the $30 \mathrm{~min}$ observation, all saline-treated dams groomed, retrieved, and grouped the pups and displayed clear active nursing. In contrast, intact morphinetreated dams did not present any maternal response. Throughout the observation period, intact morphine-treated dams kept actively exploring the cage, did not present fear-related defensive responses, and did not show any stereotypical behavior, hyperactivity, motor rigidity, or signs of sedation that would probably be expected with higher doses of morphine (Nasello et al., 1973). Likewise, all morphine-treated dams bearing unilateral NMDA lesions in the dorsal PAG also presented complete inhibition of maternal responses during the $30 \mathrm{~min}$ observation period. A histological analysis revealed that dorsal PAG lesions were, by and large, confined to the rostral half of the PAG, including both the dorsomedial and dorsolateral parts, leaving the adjacent lateral column mostly intact. In contrast, low doses of morphine failed to inhibit maternal behavior in morphine-sensitized lactating rats bearing unilateral lesions in the lateral PAG. During the 30 min observation period, all animals tested in this group presented evident active nursing $(p<0.005$ compared with intact or dorsal PAG-lesioned, morphine-treated groups). Similar to the salinetreated group, these dams also expressed other maternal responses, such as grooming, retrieving, and grouping the pups. A histological analysis revealed that lateral PAG lesions included the outer half of the lateral column at the levels of the oculomotor nucleus [a region previously shown to upregulate Fos expression in response to the morphine injection (Miranda-Paiva et al., 2003)], spreading to a variable degree over adjacent dorsolateral and ventrolateral regions. In conclusion, the results from experiment 1 indisputably show that unilateral NMDA lesions placed in the rostral lateral PAG, but not in other adjacent PAG regions, were able to block morphine-induced inhibition of maternal behavior.

In experiment 2, saline- and morphine-treated dams were tested for insect hunting only. Morphinechallenged dams started capturing the roaches significantly faster than the saline-treated group $(41.4 \pm 6.9 \mathrm{~s}$ for morphine-treated dams $\times 755.7 \pm 185.2$ $s$ for saline-treated dams; mean \pm SEM; $p=0.0017)$. Moreover, only five of the seven saline-treated dams tested consumed the five roaches during the $30 \mathrm{~min}$ observation period, taking an average time of $14 \mathrm{~min}$ (849.8 \pm $98 \mathrm{~s}$; mean \pm SEM) to consume the roaches. Conversely, all morphine-treated dams took $<10 \mathrm{~min}$ to consume the five roaches $(452 \pm 59.7 \mathrm{~s}$; mean \pm SEM $)$. The results of experiment 2 clearly suggest that morphine challenge increases the motivational drive to hunt the prey.

In experiment 3, the animals had the choice of either nursing the pups or hunting the roaches, therefore both maternal and predatory behaviors were tested. In the saline-treated group, 2 of 13 dams tested in this condition only expressed partial maternal response (i.e., mostly grooming the pups) and chose to hunt instead, consuming all five roaches placed in the cage. Three other saline-treated dams displayed both active nursing and predatory behavior. However, most of the saline-treated dams tested $(n=8)$ took care of and nursed the pups and did not attempt to catch any roach during the $30 \mathrm{~min}$ observation period (Fig. 1). In contrast, all intact morphine-treated dams tested in this condition $(n=13)$ exhibited no maternal response and showed marked predatory behavior (Fig. 1). These dams did not care for pups; instead, they vigorously sniffed around the cage and, as the prey was located, they rushed toward the roaches and tried to seize them. Compared with the saline-treated group, intact morphine-treated dams had a significantly shorter latency to capturing the first prey $(p=0.0085)$ (Fig. 1C). The dams in this group took an average time of $9 \min (564.6 \pm 61.2 \mathrm{~s}$; mean \pm SEM) to consume the five roaches.

We have further investigated how the integrity of specific columns of the PAG interferes with the expression of maternal and predatory behaviors in morphine-treated dams. Morphinetreated animals with unilateral lesions in the dorsal or ventrolateral PAG behaved similarly to the intact dams in the environment containing pups and roaches; therefore, they presented no maternal response and instead avidly hunted the roaches, showing no significant difference in latency to start capturing the prey compared with the intact morphine-treated animals (Fig. 1). In contrast, morphine-treated lactating rats with unilateral lesions in the lateral PAG behaved as if they had less interest in the prey, presenting a significant increase in the latency to start catching 
the roaches when compared with the other morphine-treated groups (i.e., intact dams and animals with unilateral lesions in the dorsal or ventrolateral PAG; $p<0.002$ for all comparisons) (Fig. 1C). Importantly, dams with unilateral lesions in the lateral PAG maintained overall hunting capability and presented no apparent deficit in catching or killing the prey. In fact, five of seven of these dams consumed all five cockroaches within the $30 \mathrm{~min}$ observation period. Although none of the dams with unilateral lesions in the lateral PAG displayed active nursing, most of them had some maternal response (Fig. 1A,B). Six of the seven dams tested in this group groomed the pups, and two of those six dams also retrieved and grouped the pups. As for experiment 1, the histological analysis revealed that the parameters used for unilateral iontophoretic NMDA deposits yielded relatively small PAG lesions with a limited rostrocaudal extension (Fig. 2). Dorsal PAG lesions tended to be confined to the rostral half of the PAG, spreading through the dorsomedial and dorsolateral parts (Fig. 2A). Ventrolateral PAG lesions were mostly confined to the caudal half of the PAG, including chiefly the ventrolateral part and extending, to a small degree, to the adjacent ventral and lateral regions (Fig. 2C). Similar to what was found in experiment 1 , lesions in the lateral PAG included the outer half of the lateral column at the levels of the oculomotor nucleus and extended, to a small degree, to the adjacent dorsolateral and ventral PAG regions (Figs. $2 B, 3 A$ ).

The results from dams with unilateral lesions in the lateral PAG were further compared with those from dams with bilateral damage in the lateral PAG. A histological analysis revealed that seven morphine-treated dams had bilateral NMDA lesions in the lateral PAG, including most of its extent at the level of the oculomotor nucleus (Fig. 3B). As shown in Figure $1 C$, bilateral damage of the rostral lateral PAG yielded a significant increase in the latency to start capturing the prey compared to unilateral lesions ( $p=$ 0.002). Most of the dams with bilateral lesions (four of seven) did not display predatory activity during the $30 \mathrm{~min}$ observation period. However, those that displayed predatory activity presented no apparent deficit in catching or killing the prey. Conversely, morphine-treated dams bearing bilateral lesions in the rostral lateral PAG presented a significant increase in active nursing compared with the other morphine-treated groups tested with pups and roaches $(p<$ 0.005 for all comparisons) (Fig. $1 A$ ). In this group, four of seven

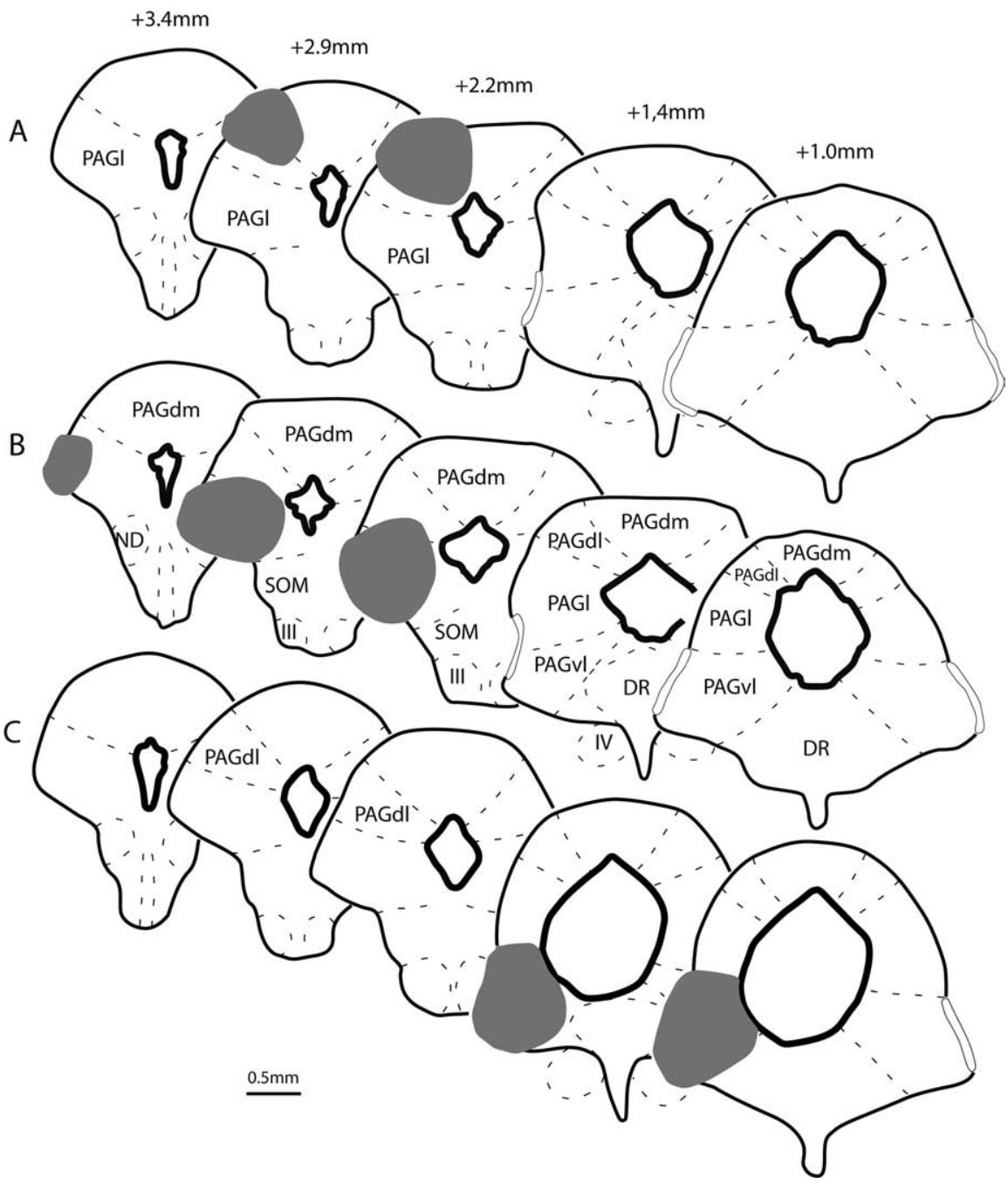

Figure 2. Experiment 3: unilateral PAG lesions. Camera lucida drawings from representative cases in experiment 3 show the total extent of unilateral PAG lesions in the dorsal $(\boldsymbol{A})$, lateral $(\boldsymbol{B})$, and ventrolateral $(\boldsymbol{C})$ PAG regions. The approximate distance from the interaural line is indicated on the top of the figures. Scale bar, $0.5 \mathrm{~mm}$. DR, Dorsal raphe nucleus; ND, nucleus of Darkschewitsch; III, oculomotor nucleus; IV, trochlear nucleus; PAGdm, dorsomedial PAG; PAGdl, dorsolateral PAG; PAGI, lateral PAG; PAGvl, ventrolateral PAG; SOM, supraoculomotor region.
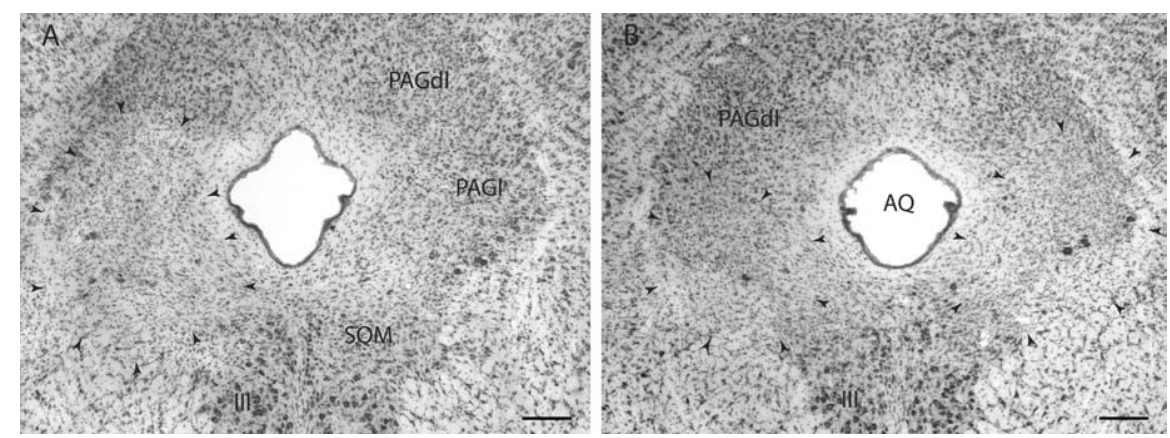

Figure 3. Experiment 3: NMDA lesion appearance. Photomicrographs of transverse thionin-stained sections, illustrating the extent and appearance of unilateral $(\boldsymbol{A})$ and bilateral $(\boldsymbol{B})$ lesions in the lateral PAG (arrowheads), from representative cases in experiment 3 are shown. Scale bars, $200 \mu \mathrm{m}$. AQ, Cerebral aqueduct; III, oculomotor nucleus; PAGdl, dorsolateral PAG; PAGI, lateral PAG; SOM, supraoculomotor region.

dams tested groomed, retrieved, and grouped the pups and displayed active nursing; one dam groomed, retrieved, and grouped the pups without presenting clear active nursing; and another dam only groomed the pups. 
In conclusion, experiment 3 indicates that maternal behavior is replaced by predatory hunting in morphine-treated dams. The current evidence suggests that the rostral lateral PAG is responsible for this behavioral shift. This conclusion is evident because unilateral lesions of the rostral lateral PAG partially impaired predatory hunting and restored part of maternal response, whereas bilateral lesions of the rostral lateral PAG produced more dramatic effects in inhibiting insect hunting and restoring maternal behavior.

\section{Discussion}

Combining Fos immunohistochemical detection and pharmacological manipulations, we had previously shown that a critical level of morphine-induced activation of the rostral lateral PAG appears to be required for the inhibition of maternal behavior produced by low doses of morphine, in previously morphinesensitized dams (Miranda-Paiva et al., 2003). In the present study, we first tested whether the rostral lateral PAG accounts for the maternal behavior inhibition using this pharmacological procedure. The results of experiment 1 indicate that unilateral NMDA lesions placed in the rostral lateral PAG, but not in other regions of the PAG, prevented the inhibition of maternal behavior with low doses of morphine in morphine-sensitized dams. These results support the view that unilateral lesions hampered the morphine-induced mobilization of the rostral lateral PAG, necessary to inhibit maternal behavior.

These results may lead to the conclusion that the rostral lateral PAG would be primarily involved in the inhibition of maternal behavior expression. However, previous studies had not supported this view, suggesting that the rostral lateral PAG appears to be only marginally involved in the expression of this behavior. In fact, lesions placed in the rostral PAG, including the rostral lateral part, did not seem to increase nursing activity (Lonstein and Stern, 1997, 1998), as would be expected for a region involved in inhibiting maternal responses.

An alternative explanation for these findings stems from previous observations that low doses of morphine induces an activation pattern of the rostral lateral PAG similar to that which occurs during predatory hunting and foraging behavior (Comoli et al., 2003; Miranda-Paiva et al., 2003). Supporting this view, in experiment 2, morphine challenge increased predatory hunting, significantly reducing the latency to start capturing the prey compared with the saline-treated group. According to our observations, the time taken to start catching the roaches appears as a useful parameter to evaluate the motivational drive to hunt the prey.

The findings from experiment 2 are compatible with the idea that predatory hunting would replace maternal behavior in response to morphine challenge. In experiment 3 , we tested this hypothesis by observing saline- and morphine-treated dams in an environment containing both pups and live cockroaches. The vast majority of the saline-treated group continued to care for the pups by actively nursing and did not attempt to catch the roaches. Conversely, morphine challenge was able to arrest the maternal responses and, at the same time, increase predatory hunting.

We further tested the possible dependence of this behavioral shift on the rostral lateral PAG. This examination revealed that the unilateral lesions spreading through this PAG region significantly increased the latency to begin catching the roaches and appeared to restore a partial maternal response. In contrast to what was just described for dams tested with pups only, when dams with unilateral lesions were tested with pups and roaches, the remaining morphine-induced activation of the rostral lateral PAG in the intact side would favor switching away from maternal to hunting behavior. This also applies for dams with no previous morphine experience, acutely challenged with low doses of morphine, presenting a relatively small degree of activation in the rostral lateral PAG (Miranda-Paiva et al., 2003). These dams do not present maternal inhibition when tested with pups only but prefer to hunt instead when tested in an environment containing pups and roaches (L. F. Felicio, unpublished observation).

Importantly, compared with intact dams and animals with unilateral PAG lesions elsewhere, animals with unilateral lesions in the rostral lateral PAG expressed less interest in the prey, whereas they continued to maintain overall hunting capabilities. Although they expressed a smaller degree of motivation for hunting the roaches, animals with unilateral lesions in the rostral lateral PAG did not show any apparent deficit in catching, killing, or eating the prey. Finally, our results indicated that the motivational drive to hunt was further decreased in animals with bilateral lesions in the rostral lateral PAG, which, in most cases, resulted in fully restoring the active nursing behavior.

Together, the present data support the idea that rostral lateral PAG activation by low doses of morphine, in previously morphine-sensitized dams, is likely to increase the motivational drive to hunt and possibly forage, therefore hampering the expression of maternal responses. By using this pharmacological procedure, we have demonstrated that the rostral lateral PAG is involved in the process of switching adaptive responses in lactating rats from maternal to predatory behavior.

At this point in our research, it is important to understand how the rostral lateral PAG relates to predatory hunting. On the afferent side, the rostral lateral PAG receives substantial inputs from the medial part of the central amygdalar nucleus (Rizvi et al., 1991). According to recent studies examining Fos immunoreactivity in rats performing predatory behavior (insect hunting), the central amygdalar nucleus serves as an important output way station for a distinct amygdalar circuit that is likely to integrate prey-related motivational values, such as prey odor and taste (Comoli et al., 2005). Furthermore, previous anterograde tracttracing studies have shown that the rostral lateral PAG receives inputs from a number of hypothalamic sites, including the retrochiamatic area (Ribeiro-Barbosa et al., 1999), the anterior part of the anterior hypothalamic nucleus (Risold et al., 1994), the anterior part of the ventromedial nucleus (Canteras et al., 1994), and the dorsal region of the dorsal premammillary nucleus (Goto et al., 2005). A great deal remains to be learned about the functional roles of these hypothalamic sites, particularly in the context of predatory behavior. Interestingly, the retrochiasmatic area has been related to the hypothalamic circuit that regulates feeding (Watts et al., 1999). The anterior part of the anterior hypothalamic nucleus, in turn, is likely to represent an important source of opioidergic inputs to the rostral lateral PAG (Harlan et al., 1987). This anterior hypothalamic site is particularly dominated by neural inputs from the septo-hippocampal districts, likely to be involved in prioritizing the temporal order of motivated responses (Risold and Swanson, 1997; Petrovich et al., 2001).

According to the present data, the rostral lateral PAG seems to influence motivational drive to chase prey and possibly forage. Information regarding the projection pattern of this PAG region should provide valuable clues toward understanding its role in predatory motivational drive. Unfortunately, our knowledge regarding the connectivity of this particular PAG region is still very fragmentary, and there is no systematic study addressing this issue (Cameron et al., 1995a,b). Preliminary tract-tracing data from our laboratory, using Phaseolus vulgaris leucoagglutinin as an anterograde tracer, revealed that one of the main targets of the 
rostral lateral PAG is the lateral hypothalamic area, where anterogradely labeled fibers were mostly distributed at tuberal levels, dorsolaterally to the fornix, primarily overlapping with a region known to contain cell groups expressing melanin-concentrating hormone $(\mathrm{MCH})$ and orexin (also known as hypocretins) (Bittencourt et al., 1992; Sakurai et al., 1998). In the context of this study, the putative relationship between the rostral lateral PAG and the MCH or orexin cells is particularly attractive. A wealth of experimental data exists to suggest that this group of neurons is associated with enhanced arousal and locomotor activity, thus potentially increasing the prey-chasing motivation, as well as the likelihood to encounter food during foraging activity (Saper et al., 2002). Additional studies are certainly needed to determine how the lateral PAG and the MCH or orexin cells interact on both anatomical and functional levels.

In conclusion, this study provides the first evidence for a previously unknown function for the PAG as a putative center for switching adaptive behavioral responses. Under natural circumstances, it is reasonable to suggest that a given behavior should be inhibited to favor the expression of another, more adaptive, response. In the present case, the morphine-challenged animals' behavior suggests that the occurrence of predatory activity was favored over maternal behavior.

Maternal behavior inhibition has also been observed during aversive situations, such as those seen in virgin females exposed to newborn pups (Sheehan et al., 2000) and dams exposed to cat odor (N. S. Canteras, unpublished observation). In these situations, a mobilization of the medial hypothalamic defensive system occurs (Sheehan et al., 2000; Dielenberg et al., 2001; Canteras, 2002). Notably, lesions of elements in this hypothalamic system, namely, the anterior or ventromedial nuclei, promote maternal response in virgin females presented with foster pups (Bridges et al., 1999; Sheehan at al., 2001) as well as advance the onset of maternal behavior in pregnant primigravid females (Mann and Babb, 2004). The mobilization of this hypothalamic system is likely to be associated with an activation of the dorsolateral PAG, seemingly responsive to psychological threats (Canteras and Goto, 1999). Therefore, under such circumstances, it would be of interest to determine the extent to which the dorsolateral PAG may account for switching from maternal to aversive responses.

To further expand the present observations, this novel role of the PAG in selecting adaptive behavioral strategies needs to be more thoroughly evaluated under other behavioral conditions. Indeed, the PAG occupies a privileged position in the CNS for accomplishing this task. On the one hand, the PAG receives a vast array of limbic information (likely to reflect the motivational status of the animal) and substantial inputs from the prefrontal cortical areas (Beitz, 1995; Floyd et al., 2000), thought to play a critical role in behavioral planning. On the other hand, the PAG is likely to influence a number of brainstem sites that are critically involved in controlling both somatomotor and automatic responses, as well as hypothalamic regions related to the control of generalized arousal and sensorimotor integration (Cameron et al., 1995a,b).

\section{References}

Bandler R, Shipley MT (1994) Columnar organization of the midbrain periaqueductal gray: modules for emotional expression. Trends Neurosci 17:379-389.

Beitz AJ (1995) Periaqueductal gray. In: The rat nervous system (Paxinos G, ed), pp173-182. Sydney: Academic.

Besson JM, Fardin V, Oliveras JL (1991) Analgesia produced by stimulation of the periaqueductal gray matter: true antinoceptive effects versus stress effects. In: The midbrain periaqueductal gray matter: functional, anatomical, and neurochemical organization (Depaulis A, Bandler R, eds) pp121-138. New York: Plenum.

Bittencourt JC, Presse F, Arias C, Peto C, Vaughan J, Nahon JL, Vale W, Sawchenko PE (1992) The melanin-concentrating hormone system of the rat brain an immuno- and hybridization histochemical characterization. J Comp Neurol 319:218-245.

Bridges RS, Grimm CT (1982) Reversal of morphine disruption of maternal behavior by concurrent treatment with the opiate antagonist naloxone. Science 218:166-168.

Bridges RS, Mann PE, Coppeta JS (1999) Hypothalamic involvement in the regulation of maternal behaviour in the rat: inhibitory roles for the ventromedial hypothalamus and the dorsal/anterior hypothalamic areas. J Neuroendocrinol 11:259-266.

Cameron AA, Khan IA, Westlund KN, Cliffer KD, Willis WD (1995a) The efferent projections of the periaqueductal gray in rat: a Phaseolus vulgarisleucoagglutinin study. I. Ascending projections. J Comp Neurol 351:568-584

Cameron AA, Khan IA, Westlund KN, Willis WD (1995b) The efferent projections of the periaqueductal gray in rat: a Phaseolus vulgarisleucoagglutinin study. II. Descending projections. J Comp Neurol 351:585-601.

Canteras NS (2002) The medial hypothalamic defensive system: hodological organization and functional implications. Pharmacol Biochem Behav 71:481-491.

Canteras NS, Goto M (1999) Fos-like immunoreactivity in the periaqueductal gray of rats exposed to a natural predator. NeuroReport 10:413-418.

Canteras NS, Simerly RB, Swanson LW (1994) Organization of projections from the ventromedial nucleus of the hypothalamus: a Phaseolus vulgarisleucoagglutinin study in the rat. J Comp Neurol 348:41-79.

Comoli E, Ribeiro-Barbosa ER, Canteras NS (2003) Predatory hunting and exposure to a live predator induce opposite patterns of Fos immunoreactivity in the PAG. Behav Brain Res 138:17-28.

Comoli E, Ribeiro-Barbosa ER, Negrão N, Goto M, Canteras NS (2005) Functional mapping of the prosencephalic systems involved in organizing predatory behavior in rats. Neuroscience 130:1055-1067.

Dielenberg RA, Hunt GE, McGregor IS (2001) "When a rat smells a cat": the distribution of c-fos expression in rat brain following exposure to a predator odor. Neuroscience 104:1085-1097.

Eisenberg JF, Leyhausen P (1972) The phylogenesis of predatory behavior in mammals. Z Tierpsychol 30:59-93.

Floyd NS, Price JL, Ferry AT, Keay KA, Bandler R (2000) Orbitomedial prefrontal cortical projections to distinct longitudinal columns of the periaqueductal gray in the rat. J Comp Neurol 422:556-578.

Goto M, Canteras NS, Burns G, Swanson LW (2005) Projections from the subfornical region of the lateral hypothalamic area. J Comp Neurol 493:412-438.

Grimm CT, Bridges RS (1983) Opiate regulation of maternal behavior in the rat. Pharmacol Biochem Behav 19:609-616.

Harlan RE, Shivers BD, Romano GJ, Howells RD, Pfaff DW (1987) Localization of preproenkephalin mRNA in the rat brain and spinal cord by in situ hybridization. J Comp Neurol 258:159-184.

Heeb MM, Yahr P (2000) Cell-body lesions of the posterodorsal preoptic nucleus or posterodorsal medial amygdala, but not the parvicellular subparafascicular thalamus, disrupt mating in male gerbils. Physiol Behav 68:317-331.

Jürgens U (1994) The role of the periaqueductal grey in vocal behavior. Behav Brain Res 62:107-117.

Keay KA, Bundler R (2001) Parallel circuits mediating distinct emotional coping reactions to different types of stress. Neurosci Biobehav Rev 25:669-678.

Lonstein JS, Stern JM (1997) Role of the midbrain periaqueductal gray in maternal nurturance and aggression: c-fos and electrolytic lesion studies in lactating rats. J Neurosci 17:3364-3378.

Lonstein JS, Stern JM (1998) Site and behavioral specificity of periaqueductal gray lesions on postpartum sexual, maternal, and aggressive behaviors in rats. Brain Res 804:21-35.

Lovick TA (1993) Integrated activity of cardiovascular and pain regulatory sytems: role in adaptive behavioral responses. Prog Neurobiol 40:631-644.

Mann PE, Babb JA (2004) Disinhibition of maternal behavior following 
neurotoxic lesions of the hypothalamus in primigravid rats. Brain Res 1025:51-58.

Mann PE, Kinsley CH, Bridges RS (1991) Opioid receptor subtype involvement in maternal behavior in lactating rats. Neuroendocrinology 53:487-492.

Miranda-Paiva CM, Ribeiro-Barbosa ER, Canteras NS, Felicio LF (2003) A role for the periaqueductal grey in opioidergic inhibition of maternal behaviour. Eur J Neurosci 18:667-674.

Nasello AG, Tannhauser R, Izquierdo I (1973) Effect of morphine on the RNA and ATP concentration of brain structures of the rat. Pharmacology 10:56-59.

Petrovich GD, Canteras NS, Swanson LW (2001) Combinatorial amygdalar inputs to hippocampal domains and hypothalamic behavior systems. Brain Res Rev 38:247-289.

Ribeiro-Barbosa ER, Skorupa AL, Cipolla-Neto J, Canteras NS (1999) Projections of the basal retrochiasmatic area: a neural site involved in the photic control of pineal metabolism. Brain Res 839:35-40.

Risold PY, Swanson LW (1997) Connections of the rat lateral septal complex. Brain Res Rev 24:115-195.

Risold PY, Canteras NS, Swanson LW (1994) Organization of projections from the anterior hypothalamic nucleus: a Phaseolus vulgarisleucoagglutinin study in the rat. J Comp Neurol 348:1-40.

Rizvi TA, Ennis M, Behbehani MM, Shipley MT (1991) Connections between the central nucleus of the amygdala and the midbrain periaqueductal gray: topography and reciprocity. J Comp Neurol 303:121-131.

Rubin BS, Bridges RS (1984) Disruption of ongoing maternal behavior re- sponsiveness in rats by central administration of morphine sulphate. Brain Res 307:91-97.

Sakuma Y, Paff DW (1979) Facilitation of female reproductive behavior from mesencephalic central gray in the rat. Am J Physiol 237:R278-R284.

Sakurai T, Amemiya A, Ishii M, Matsuzaki I, Chemelli RM, Tanaka H, Williams SC, Richardson JA, Kozlowski GP, Wilson S, Arch JR, Buckingham RE, Haynes AC, Carr SA, Annan RS, McNulty DE, Liu WS, Terrett JA, Elshourbagy NA, Bergsma DJ, et al. (1998) Orexins and orexin receptors a family of hypothalamic neuropeptides and $\mathrm{G}$ protein-coupled receptors that regulate feeding behavior. Cell 92:573-585.

Saper CB, Chou TC, Elmquist JK (2002) The need to feed: homeostatic and hedonic control of eating. Neuron 36:199-211.

Sheehan TP, Cirrito J, Numan MJ, Numan M (2000) Using c-Fos immunocytochemistry to identify forebrain regions that may inhibit maternal behavior in rats. Behav Neurosci 114:337-352.

Sheehan TP, Paul M, Amaral E, Numan MJ, Numan M (2001) Evidence that the medial amygdala projects to the anterior/ventromedial hypothalamic nuclei to inhibit maternal behavior in rats. Neuroscience 106:341-356.

Sheskin DJ (2000) Handbook of parmetric and nonparametric statiscal procedures. Boca Raton, FL: Chapman and Hall/CRC.

Slamberova R, Bar N, Vathy I (2003) Long-term effects of prenatal morphine exposure on maternal behaviors differ from the effects of direct chronic morphine treatment. Dev Psychobiol 43:281-289.

Watts AG, Sanchez-Watts G, Kelly AB (1999) Distinct patterns of neuropeptide gene expression in the lateral hypothalamic area and arcuate nucleus are associated with dehydration-induced anorexia. J Neurosci 19:6111-6121. 